\title{
Geographie in einer verstädterten Gemeinschaft
}

Walter Leimgruber

Das Problem der Verstädterung beschäftigt heutzutage die Geographen in aller Welt. Um einmal auch den Menschen als Objekt der Verstädterung genauer zu betrachten, stellte die britische Geographical Association ihre letztjährige Konferenz unter das Leitwort "Geography in an urbanized Society». Die Tagung, die in den modernen Gebäulichkeiten der University of Sussex bei Brighton stattfand, wurde von der Sektion Brighton der Geographical Association in Zusammenarbeit mit den Geographen der Universität organisiert. Aus dem vielseitigen Programm seien hier nur einige wesentliche Punkte herausgegriffen. Dabei muß man sich im klaren sein, daß alle Aussagen auf den spezifischen englischen Verhältnissen beruhen und deshalb nicht ohne weiteres auf den Kontinent oder gar die Schweiz übertragen werden können.

In seinem Eröffnungsreferat über "The Shadow of the Metropolis" steckte Prof. T. E. Elkins, Vorsteher des Geographischen Instituts der University of Sussex, den Problemkreis ab, indem er die Entwicklung der Südküste - Brightons im speziellen - und des Gebietes zwischen der Küste und London betrachtete. War Brighton anfänglich nur ein Ferienort für die Oberschicht, so fand mit dem Aufkommen der Eisenbahn auch der Mittelstand den Weg an die Südküste. Allerdings entwickelten sich die Siedlungen nur punkthaft und sehr langsam. Entlang der Bahnlinie entstanden nördlich der South Downs bereits die ersten Schlaforte für Pendler nach London. Die eigentliche Umwälzung aber brachte das Auto. Die Siedlungen an der Küste wuchsen immer rascher; sie erstrecken sich heute als fast ununterbrochenes Band von Brighton über Worthing nach Littlehampton. Schon früh erkannten Private und Behörden, daß aktiver Landschaftsschutz unumgänglich war, wollte man den Sonnenhang der South Downs vor der völligen Überbauung bewahren. In den Dreißiger Jahren wurde die 90-m-(300$\mathrm{ft}-)$ Höhenlinie als obere Grenze für die Siedlungsausdehnung bestimmt. Sie gilt - mit kleineren, lokal bedingten Abweichungen - heute noch und bildet eine äußerst markante Linie im Landschaftsbild. Die Nähe der Metropole macht sich aber noch in anderer Hinsicht bemerkbar:

1. Brighton kann heute mit der Bahn von Victoria Station (London) aus in knapp einer Stunde erreicht werden. Das ist ein Grund, weshalb es heute nicht mehr vorwiegend ein Ferienort ist, sondern vor allem von Tages- und WochenendAusflüglern - allerdings in großer Zahl - aufgesucht wird. Auch werden immer häufiger Konferenzen hier abgehalten.

2. Die Nähe Londons hat auch zu einer kleinen Ansiedlung von Industrie geführt, wodurch die Abhängigkeit vom Tourismus mit seinem jahreszeitlichen Rhythmus nicht so groß ist.

3. Immer mehr Leute übersiedeln aus London an die Südküste, hier ihren Ruhestand zu verbringen. Die Altersstruktur Brightons hat sich 1961 gegenüber 1901 stark verändert: der Anteil der oberen Altersklassen ist beträchtlich angestiegen, aus einer Pyramide ist eine Glocke geworden.

4. Nördlich der Downs wird das Bild vor allem von der Pendelwanderung nach London beherrscht. Die Altersstruktur der Zuwanderer in diesem Raum ist völlig verschieden von jener an der Südküste: es sind in erster Linie junge Leute, die sich hier niederlassen.

Mit dem soziologischen Aspekt befaßten sich in ihren Referaten Dr. D. E. C. Eversley und Mr. B. T. Wood von der University of Sussex. Dr. Eversley hob vor allem zwei Punkte hervor, die vermehrter Beachtung bedürfen:

1. Beim Studium von Bevölkerungswanderungen ist die absolute Zahl nicht entscheidend. Die Wirtschaftsstruktur einer Region hängt zu einem ebenso großen oder noch größeren Teil von Alter, Geschlecht und Handfertigkeitsgrad der Bevölkerung ab.

2. Mit zunehmendem Einkommen und zunehmender Freizeit nimmt das Raumbedürfnis zu, und zwar nicht proportional zur Bevölkerung, sondern proportional zu der Zahl unabhängiger Haushalte plus Anteil der Zunahme der verfügbaren Freizeit plus Anteil der Zunahme des nicht unbedingt lebensnotwendigen Bedarfs.

In einem Land, in welchem der Grundsatz "My house is my castle» gilt, erscheint Punkt 2 als besonders wichtig, da sich gerade seinetwegen besondere Raumprobleme stellen.

Mr. Wood stellte die alten Wohnquartiere in den Städten - gekennzeichnet durch niedrige Grundstücksteuern und ein enges Netz von persönlichen 
Beziehungen - den neuen Wohnquartieren in den New Towns gegenüber, die sich in höhern Grundstücksteuern und der Abwesenheit des eingespielten Netzes von Beziehungen offenbaren. In solchen neuen Quartieren muß dieses Netz zuerst hergestellt werden.

Die Abwanderung der Bevölkerung aus London zum Teil auf privater Basis, zum Teil als Folge einer offiziellen Politik, Londons Bevölkerung bis in die achtziger Jahre auf sieben Millionen zu verringern - und die geplante Neuentwicklung in der "Outer Metropolitan Area» führen zu Pendlerproblemen, die Mr. A. Todd von der University of Kent skizzierte. Viele Leute suchen außerhalb Londons ein Haus und einen Arbeitsplatz. Da sich indessen Planung und freier Wille des Menschen nicht immer decken, kann es vorkommen, daß Wohn- und Arbeitsort noch weiter auseinander liegen als vor dem Wechsel. Der Pendler legt unter Umständen beträchtliche Stecken zurück, was ihn und den Staat ziemlich viel Geld kostet.

Ein von Dr. J. R. Blunden, University of Sussex, geleitetes Symposium befaßte sich besonders mit der Auswirkung der Stadt auf die Landwirtschaft. Einige Studien, die im Südosten, im Einflußbereich Londons, durchgeführt worden waren, wurden verglichen und zeigten alle ein ähnliches Bild. Die Zahl der hauptberuflich betriebenen Farmen nimmt ständig ab, vor allem in der Nähe der großen Verkehrslinien. Dafür nimmt die bearbeitete Fläche je Betrieb zu. Die Zahl der nebenberuflichen Landwirte ist zum Teil gewaltig angestiegen. Es sind unter anderem Leute mit hohen Stellungen in der City - Bankiers, Managers usw. -, die sich auf dem Land einen Bauernhof erstehen und einen kapitalintensiven Betrieb einrichten, bei gleichzeitiger Spezialisierung auf ein bis zwei Produkte. Der traditio- nelle Bauer wird dadurch gezwungen, seinerseits auch mehr Kapital zu investieren, und dabei verschwinden Klein- und auch Mittelbetriebe. Die Gegensätze sind oft recht nahe beieinander. In der Umgebung von Haywards Heath $(20 \mathrm{~km} \mathrm{~N}$ Brightons) sind $95 \%$ aller Farmen nebenberuflich. In Henfield (15 km NW Brightons) sind es ihrer nur $25 \%$. Auf den South Downs werden praktisch alle Betriebe hauptberuflich betrieben. Je mehr Berufsbauern aber eine Gegend zählt, um so komplizierter ist auch die gesamte Betriebsstruktur.

Über die Entwicklung von Crawley New Town sprach Mr. D. Stoppard. Die Stadt ist durch eine recht junge Altersstruktur gekennzeichnet. Den Haupterwerbszweig der Beschäftigten bildet die Industrie. Doch ist das gegenwärtige Industrieareal noch nicht so groß, wie es im Plan vorgesehen war. Trotzdem hat die Industrie zahlreiche Zuwanderer angelockt, und bereits mußte eine neue, im Plan nicht vorgesehene Nachbarsiedlung angelegt werden. Das Wachstum wurde unter anderem durch den Ausbau des Flughafens Gatwick beschleunigt. Sehr bald wird die weitere Entwicklung der Stadt jenseits der administrativen Grenzen weitergehen. So entsteht allmählich eine "Subregion»; sie bietet die einzige Möglichkeit zur Lösung der sich stellenden Probleme - vor allem jenem der Arbeitskräfte. An diesem Beispiel hat sich die Idee einer sich selbst erhaltenden Stadt als nicht durchführbar erwiesen.

Die Exkursionen waren so angelegt, daß jede eine willkommene Ergänzung zu den Themen der Referate und Symposien bot. Insgesamt vermittelte die Konferenz ein gutes Bild von der Arbeit britischer Geographen in einem Bereich, der auch für unser Land von immer größerer Bedeutung wird. 